\title{
Calcium-activated potassium channels as potential early markers of human cervical cancer
}

\author{
ANA RAMÍREZ ${ }^{1}$, EUNICE VERA ${ }^{1}$, ARMANDO GAMBOA-DOMÍNGUEZ ${ }^{2}$, \\ PAUL LAMBERT $^{3}$, PATRICIO GARIGLIO ${ }^{4}$ and JAVIER CAMACHO ${ }^{1}$
}

\author{
${ }^{1}$ Department of Pharmacology, Centro de Investigación y de Estudios Avanzados del Instituto Politécnico Nacional, \\ Mexico City 07360; 'Departamento de Patología, Instituto Nacional de Ciencias Médicas y Nutrición 'Salvador Zubirán', \\ Mexico City 14000, Mexico; ${ }^{3}$ McArdle Laboratory for Cancer Research, University of Wisconsin School of Medicine \\ and Public Health, Madison, WI 53706, USA; ${ }^{4}$ Department of Genetics and Molecular Biology, Centro de \\ Investigación y de Estudios Avanzados del Instituto Politécnico Nacional, Mexico City 07360, Mexico
}

Received October 11, 2017; Accepted January 18, 2018

DOI: $10.3892 / \mathrm{ol} .2018 .8187$

\begin{abstract}
Cervical cancer is a major cause of cancer-associated mortality in women in developing countries. Thus, novel early markers are required. Ion channels have gained great interest as tumor markers, including cervical cancer. The calcium-activated potassium channel KCNMA1 (subunit $\alpha-1$ from subfamily $\mathrm{M}$ ) has been associated with different malignancies, including tumors such as breast and ovarian cancer that are influenced by hormones. The KCNMA1 channel blocker iberiotoxin decreases the proliferation of HeLa cervical cancer cells. Nevertheless, KCNMA1 channel expression during cervical carcinogenesis remains elusive. Therefore, KCNMA1 expression was studied in cervical cancer development. FVB transgenic mice expressing the E7-oncogene of high-risk human papilloma virus, and non-transgenic mice were treated with estradiol-releasing pellets during 3 or 6 months to induce cervical lesions. Twenty-four human cervical biopsies from non-cancerous, low- or high-grade intraepithelial lesions, or cervical cancer were also studied. mRNA and protein expression was assessed by reverse transcription-quantitative polymerase chain reaction and immunohistochemistry, respectively. Cervical dysplasia and carcinoma were observed only in the transgenic mice treated with estradiol for 3 and 6 months, respectively. Estradiol treatment increased KCNMA1 mRNA and protein expression in all groups; however, the highest levels were observed in the transgenic mice with carcinoma. KCNMA1 protein expression in the squamous cells of the transformation zone was observed only in the transgenic mice with cervical dysplasia or cancer.
\end{abstract}

Correspondence to: Dr Javier Camacho, Department of Pharmacology, Centro de Investigación y de Estudios Avanzados del Instituto Politécnico Nacional, 2508 Avenida Instituto Politécnico Nacional, Mexico City 07360, Mexico

E-mail: fcamacho@cinvestav.mx

Key words: calcium-activated potassium channels, cervical cancer, human papilloma virus, estrogens, ether à-go-go-1 channels
Human biopsies from non-cancerous cervix did not display KCNMA1 protein expression; in contrast, the majority of the tissues with cervical lesions (16/18) displayed KCNMA1 protein expression. The lowest channel immunostaining intensity was observed in biopsies from low-grade dysplasia and the strongest in the carcinoma tissues. These results suggest KCNMA1 channels as potential early cervical cancer markers.

\section{Introduction}

Cervical cancer is a major leading cause of cancer death in women worldwide, especially in developing countries $(1,2)$. Thus, novel early markers, as well as new therapeutic alternatives, are urgently needed. Precancerous lesions of cervical cancer begin with low squamous intraepithelial lesions that develop into high squamous intraepithelial lesions, and then into squamous cell carcinoma, which is the most common type of cervical cancer. Persistent infection with HR-HPV is the main risk factor for the development of cervical carcinoma. However, HR-HPV infection is necessary but not sufficient to cause cervical cancer, and $90 \%$ of the women infected with HR-HPV will be cleared within 1-2 years (1). Thus, other factors including genetic predisposition, smoking, environment, and chronic estrogen exposure have been suggested to participate in the development of cervical cancer $(3,4)$. Actually, over-expression of the enzyme aromatase correlates with cervical carcinoma progression (5), and in vivo studies with transgenic mice expressing the E7-oncogene of HPV16 showed that estrogen treatment is necessary for cervical carcinogenesis (6).

Several ion channels have been proposed as novel tools in oncology as potential tumor markers and therapeutic targets (7). Potassium channels are the most diverse class of ion channels, and have been associated with different aspects of cancer including tumor cell proliferation, migration, invasion, and angiogenesis (8). The calcium-activated potassium channels KCNMA1 $\left(\mathrm{K}_{\mathrm{Ca}} 1.1, \mathrm{BK}\right)$ are expressed throughout the body and are activated by membrane depolarization and/or by intracellular $\mathrm{Ca}^{+2}(9,10)$. These channels have been involved in different functions relevant in cancer including cell 
proliferation and migration (11). Actually, KCNMA1 channels are over-expressed in diverse cancers including glioma (12), as well as tumors influenced by hormones like breast, ovarian and prostate $(13,14)$, among others. In addition, inhibition of channel expression by antisense probes or channel blockage with different drugs, decreases estradiol-induced activation of the channel and cell growth in breast (13) and prostate cancer (14), respectively. In cervical cancer it has been suggested the presence of KCNMA1-mediated potassium currents in HeLa cervical cancer cells, and it has been also shown that iberiotoxin (a selective KCNMA1 channel blocker) decreases HeLa cell proliferation (15). However, the expression of this channel during cervical carcinogenesis, and its expression in cervical cancer and pre-malignant stages in human samples remain unknown. Therefore, here we studied KCNMA1 expression in cervical cancer development in vivo, as well as in human cervical biopsies from normal, pre-malignant and carcinoma tissues. The results strongly suggest KCNMA1 channel as a potential early cervical cancer marker.

\section{Materials and methods}

Transgenic mice and hormone treatment. We used the FVB non-transgenic and the FVB-K14E7 transgenic mice expressing the E7-HPV16 oncogene, previously described (16). The E7-HPV16 oncogenic expression is directed to the basal cells of the squamous epithelium under the control of the human keratin 14 transcriptional promoter. All the animals were housed in accordance with the institutional protocols and the Guide for the Care and Use of Laboratory Animal. The mice were treated according to the protocol 0452-09 approved by the Research Unit for Laboratory Animal Care Committee (UPEAL-CINVESTAV, Mexico City, Mexico), and housed with a photoperiod of $12 \mathrm{~h}$ of light under pathogen-free conditions. Fifty-six virgin females were used. A total of 14 FVB and 14 K14E7 mice at 4-6 weeks old were given continuous-release pellets delivering $17 \beta$-estradiol (E2) (0.05 mg/60 days, Innovative Research of America, Sarasota, FL). The pellets were subcutaneously inserted under the dorsal skin every 60 days. The mice were sacrificed by cervical dislocation at either 4 months old [3 months of estradiol treatment, weighing $28.6 \pm 2.2 \mathrm{~g}$ (mean $\pm \mathrm{s}$. d)] or 7 months old [6 months of estradiol treatment, weighing $32.7 \pm 3.7 \mathrm{~g}$ (mean $\pm \mathrm{s}$. d.)]. The rest of the animals (14 K14E7 and $14 \mathrm{FVB}$ mice) of the same age were left untreated as controls.

Human biopsies. Tissue samples from non-cancerous cervix, low-grade cervical dysplasia, high-grade cervical dysplasia and cervical cancer were retrospectively collected from the Pathology Department of the Instituto Nacional de Nutrición y Ciencias Médicas Salvador Zubirán (Mexico City, Mexico). Six tissue samples of each condition (total=24 samples) were studied for KCNMA1 protein expression by immunohistochemistry.

Immunohistochemistry. The female reproductive tracts of the mice were obtained by surgery, fixed in $4 \%$ paraformaldehyde, and embedded in paraffin. Tissue sections $(3 \mu \mathrm{m})$ were collected and deparaffinized in xylene, rehydrated with decreasing graded concentrations of ethanol, and stained with hematoxylin and eosin. The hystopathological analysis was performed based on morphological criteria for transgenic mouse cervical squamous carcinogenesis (17). Human and mice tissue sections were processed for immunohistochemical staining using the rabbit poly-detector HRP/DAB detection system (Bio-SB, Inc., Santa Barbara, CA, USA) according to manufacturer's recommendations. After deparaffinization, the slides were treated with citrate buffer ( $\mathrm{pH} 6,15 \mathrm{~min}$ ) for heat-induced antigen retrieval, then treated for $10 \mathrm{~min}$ with peroxidase blocker, and incubated for $2 \mathrm{~h}$ with the anti-KCNMA1 rabbit polyclonal antibody (1:250, cat. no. Ab3586; Abcam Cambridge, UK). Secondary detection was performed with the goat anti-rabbit biotinylated IgG followed by Streptavidin HRP and visualized with DAB. All slides were counterstained with hematoxylin (Dako; Agilent Technologies, Inc., Santa Clara, CA, USA), dehydrated with increasing graded concentrations of ethanol, and mounted with permanent mounting media. Images were captured using an Olympus IX51 microscope, Olympus DP70 camera (Olympus Corporation, Tokyo, Japan). The presence of the channel protein was detected as brown immunostaining. For the human sections, staining intensity was scored on an arbitrary scale as either $(-)$ negative, $(+)$ weak, $(++)$ moderate, or $(+++)$ intense immunoreactivity.

Gene expression analysis by real-time RT-qPCR. Total RNA was extracted using TRIzol reagent (Sigma-Aldrich; Merck KGaA, Darmstadt, Germany). The RNA concentration and quality were determined by spectrophotometry (NanoDrop 1000; Thermo Fisher Scientific, Inc., Wilmington, DE, USA), and $5 \mu \mathrm{g}$ of RNA were used to synthesize cDNA using the M-MuLV reverse transcriptase (New England Biolabs, Ipswich, MA, USA). RT-qPCR was performed using the Step-One Plus Real-Time PCR system (Applied Biosystems; Thermo Fisher Scientific, Inc., Waltham, MA, USA) with TaqMan ${ }^{\circledR}$ universal PCR master mix (Applied Biosystems; Thermo Fisher Scientific, Inc.), and Kcnmal and Kcnh1 TaqMan ${ }^{\circledR}$ probes (cat nos. Mm00516078_m1 and Mm01316769_m1, respectively); cyclophilin was used as constitutive gene (cat no. Mm03302254_g1). Each duplicate was set up in a total reaction volume of $12.5 \mu 1$ [ $1 \mu \mathrm{l}$ cDNA, $6.3 \mu 1$ master mix and $0.63 \mu \mathrm{l}$ of probe $(20 \mathrm{X})$ and water]. The thermal cycling conditions were: $95^{\circ} \mathrm{C}$ for $10 \mathrm{~min}$ for activation of Taq DNA polymerase and 40 cycles of PCR were performed. Each cycle consisted of $95^{\circ} \mathrm{C}$ for $15 \mathrm{sec}$ for melting and $60^{\circ} \mathrm{C}$ for $1 \mathrm{~min}$ for annealing and extension. Each plate contained no template control (NTC) for each probe, a target reagent sample (carried up during all the process), a negative sample (no reverse transcriptase enzyme), and duplicates for each cDNA. Data were analyzed by the $\Delta \Delta \mathrm{Cq}$ method (18).

Statistical analysis. All data are presented as mean \pm standard deviation (SD) and were analyzed by one-way analysis of variance (ANOVA) followed by the Tukey test. Differences were considered significant when $\mathrm{P}<0.05$. The analysis was performed with the Graphpad Prism Software (Version 5; GraphPad Software, Inc., La Jolla, CA, USA).

\section{Results}

The transgenic mice as a suitable in vivo model to study channel expression. Gene expression profiles have been studied 
A

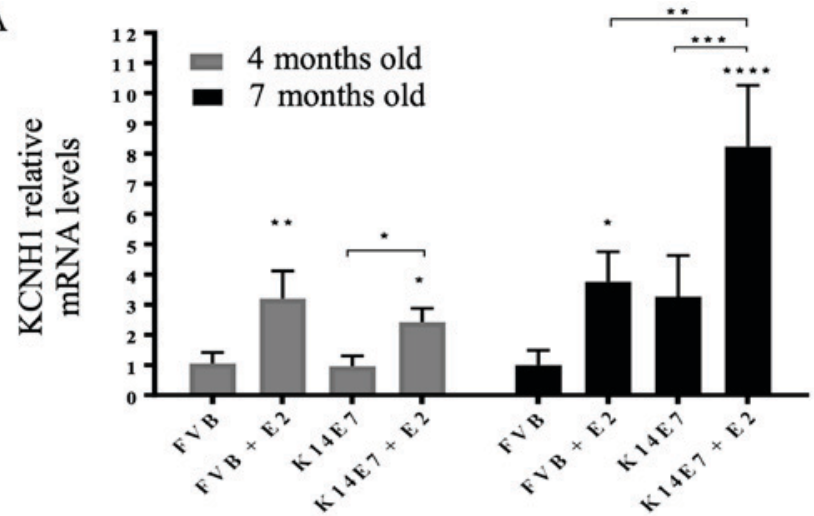

B a FVB
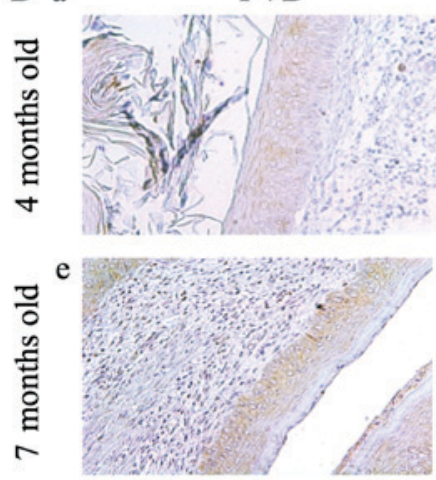

K14E7
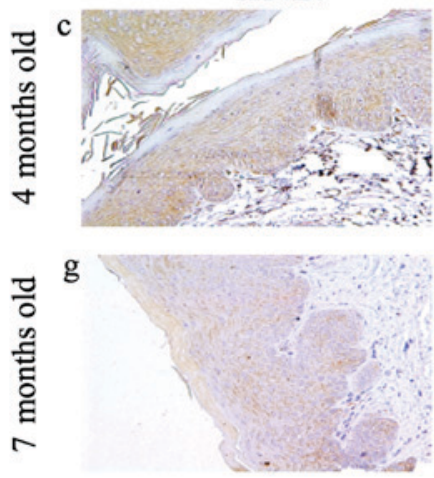

b

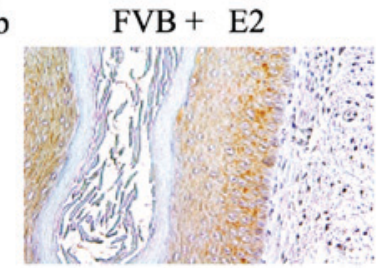

$\mathrm{f}$

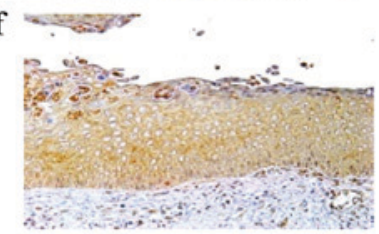

$\mathrm{K} 14 \mathrm{E} 7+\mathrm{E} 2$

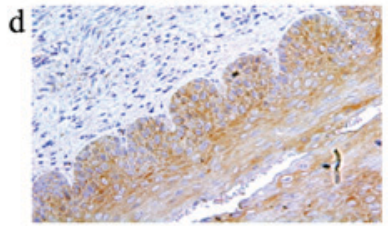

$\mathrm{h}$

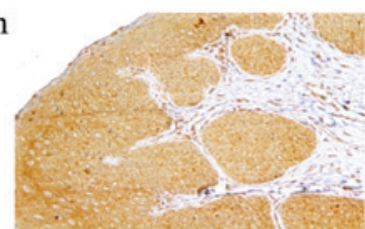

Figure 1. Eag1 channels are up-regulated by estradiol, and in cervical cancer. (A) 17ß-estradiol (E2) enhances Kcnh1 mRNA expression in the cervix of non-transgenic (FVB) and transgenic (K14E7) mice, but the highest levels were found in the cervical cancer group (K14E7 + E2, 7 months old). Four independent experiments (mean $\pm \mathrm{SD}$ ), ${ }^{*} \mathrm{P}<0.05,{ }^{* * *} \mathrm{P}<0.005,{ }^{* * * *} \mathrm{P}<0.001$, and ${ }^{* * * * *} \mathrm{P}<0.0001$ vs. the corresponding FVB group, unless indicated. (B) Protein expression was studied by immunohistochemistry in 4-month-old (a-d) and 7-month-old (e-h) mice. The presence of the protein is revealed as brown immunostaining, $\mathrm{n}=3$ mice per group. Magnification, $\mathrm{x} 20$.

in the K14E7 transgenic mice $(19,20)$. However, no mRNA and protein expression of ion channels have been described in this in vivo model. We first wondered if this model resembles the in vitro and clinical observations regarding other potassium channel proposed as an early cervical cancer marker. Ether-á-go-go-1 (Kcnh1, Kv10.1, Eag1) potassium channels have been suggested as early cervical cancer markers because their expression is regulated by HPV oncogenes and estradiol, and because their protein expression increases from normal cervix to intraepithelial lesions and cervical carcinomas in human biopsies and cervical cytologies $(21,22)$. Fig. 1 shows that KCNH1 mRNA and protein expression increases with

estradiol treatment; the highest mRNA expression is observed in the transgenic mice with cervical cancer (Fig. 1A). In the histological analysis we observed that non-transgenic FVB mice showed normal cervix (Fig. 1Ba and Be) and very weak channel immunostaining, but presented cervical hyperplasia when treated with estradiol for 3 or 6 months, as well as a higher protein expression (brown inmunostaining (Fig. 1Bb and Bf). In contrast, transgenic K14E7 mice displayed low-grade cervical dysplasia or cervical cancer when treated with estradiol for 3 or 6 months, respectively (Fig. 1Bd and Bh); the strongest KCNH1 immunostaining was observed in the cervical cancer mice. This in vivo model resembles the in vitro and clinical observations reported for $\mathrm{KCNH} 1$ channels, which suggest it as a representative model to study ion channels as early cervical cancer markers. Then, we looked for KCNMA1 channel expression.

Differential expression of KCNMA1 channel during cervical carcinogenesis in mice. Fig. 2A shows that E2 increased Kcnmal mRNA levels in all groups. However, the highest expression corresponded to the cervical cancer group, that is, to the transgenic mice treated with $17 \beta$-estradiol for 6 months (7 months old). This up-regulation was also observed at the protein level (Fig. 2B). The strongest KCNMA1 immunostaining was observed in the transgenic groups displaying either cervical dysplasia (Fig. 2Bf), or cancer (Fig. 2Bh).

Expression of KCMA1 channels in the cervical transformation zone in mice. The cervical transformation zone holds different cell types and is the region where most of the abnormal changes occur in human cervical cancer, and where the progenitor cell type for cervical cancer are supposed to reside. Interestingly, we observed KCNMA1 protein expression in the endocervical epithelium of the transformation zone in all the groups (Fig. 2C). However, immunostaining in the squamous epithelium was only observed in the transgenic mice treated with $17 \beta$-estradiol, that is, those with either premalignant cervical lesion (Fig. 2Cf), or with cervical cancer where this immunostaining was increased (Fig. 2Ch).

KCNMA1 protein expression in human cervical tissues. Next, we investigated KCNMA1 protein expression in various human cervical biopsies from different histological tumor grades. KCNMA1 expression was not detectable in the cervical epithelium of normal (non-cancerous) cervical tissues (Fig. 3). In contrast, most of the tissues with cervical lesions displayed protein expression (16/18). The percentages of KCNMA1-positive human biopsies were $0 \%$ ( 0 out of 6 ) for non-cancerous cervix, 66\% (4 out of 6 ) for low-grade intraepithelial lesions, and $100 \%$ for high-grade intraepithelial lesions (6 out of 6 ) and cervical carcinoma (6 out of 6 ). The lowest channel immunostaining intensity was observed in the biopsies from low-grade dysplasia, and the strongest intensity in the carcinoma tissues.

\section{Discussion}

Several potassium channels have been associated with different types of cancer. However, there are only few reports studying the expression of ion channels as early cancer markers in 


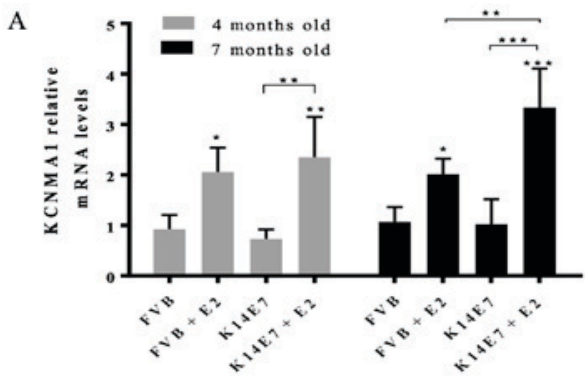

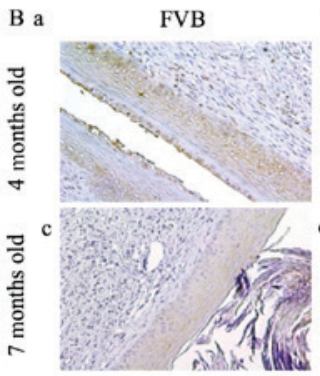

K14E7

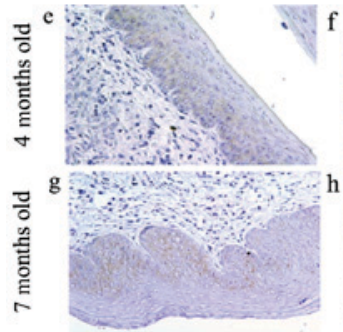

b $\quad \mathrm{FVB}+\mathrm{E} 2$

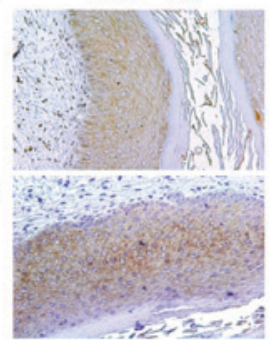

$\mathrm{K} 14 \mathrm{E} 7+\mathrm{E} 2$

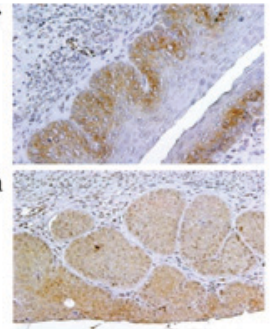

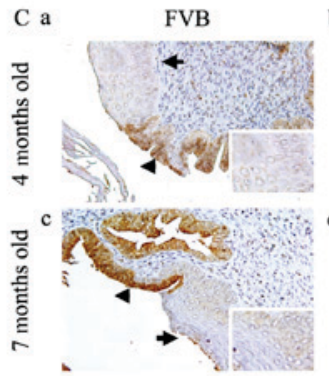

K14E7

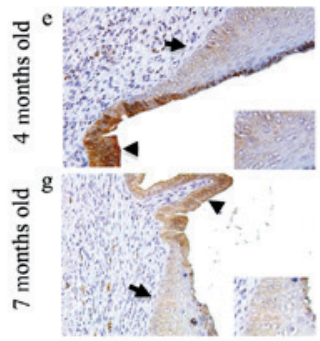

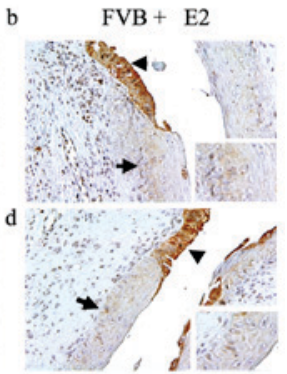

$\mathrm{K} 14 \mathrm{E} 7+\mathrm{E} 2$

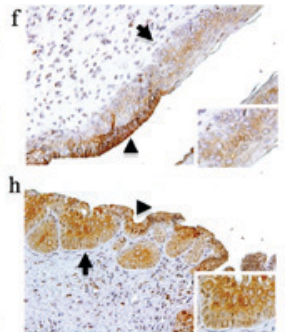

Figure 2. Increased KCNMA1 expression in cervical dysplasia and cancer in the K14E7 transgenic mice. (A) Gene expression of Kcnma1 mRNA was enhanced by $17 \beta$-estradiol in all the groups, but the highest levels were found in the cervical cancer group, that is, in the transgenic mice treated with E2 for 6 months ( 7 months old). Four independent experiments (mean $\pm \mathrm{SD}$ ), ${ }^{*} \mathrm{P}<0.05,{ }^{* *} \mathrm{P}<0.005$, and ${ }^{* * *} \mathrm{P}<0.001$ vs. the corresponding FVB group, unless indicated. (B) KCNMA1 Protein expression (brown immunostaining) was increased throughout the multiple layers of cells in low-grade cervical dysplasia (f), and cervical cancer (h) in the transgenic mice, while weaker protein expression was detected in either the normal cervical squamous epithelium (a and c) or hyperplastic lesions (b and d) of non-transgenic FVB mice, and in the cervical squamous epithelium of transgenic mice non-treated with E2 (e and g), (C) Representative images of the cervical transformation zone showing KCNMA1 protein expression in the endocervical epithelium (black triangles) of all groups. Interestingly, the squamous cells (arrows, and inserts at the right bottom side of each panel) displayed positive immunostaining only in the transgenic mice with either cervical dysplasia (f) or cervical cancer (h). $n=3$ mice per group. Magnification, $x 20$.
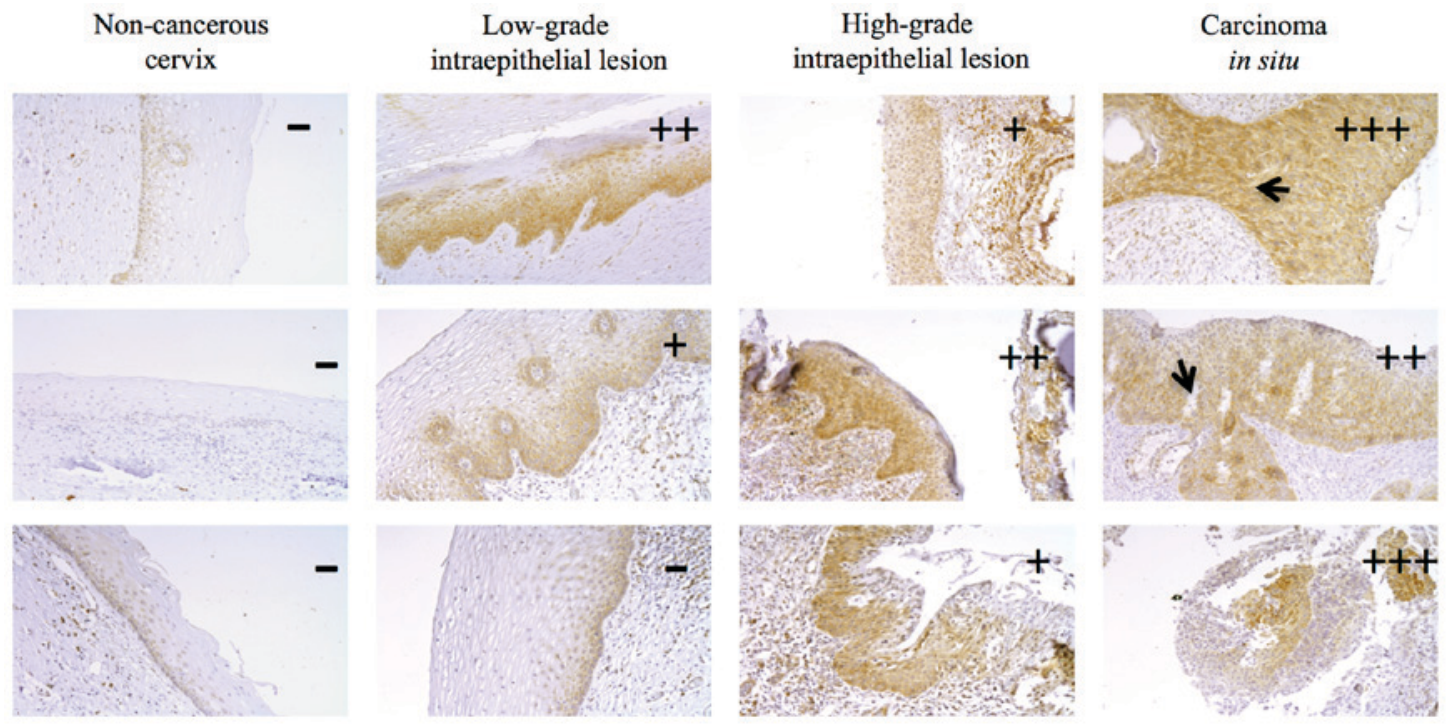

Figure 3. KCNMA1 protein expression in human cervical dysplasia and carcinoma. The images show variable immunostaining intensity of KCNMA1 protein expression (brown immunostaining). Non-cancerous human biopsies were negative for KCNMA1 immunostaining. Low-grade cervical dysplasia samples displayed the lowest intensity, while the stronger immunostaining was observed in the carcinoma in situ biopsies. The staining intensity was scored as indicated under Materials and methods. The arrows indicate the rupture of the basal membrane, probably caused by the proliferation of cervical cancer cells. Representative images from six independent experiments in each condition are shown. Magnification, x20. 
premalignant lesions $(7,8)$. Here, we show for the first time that KCNMA1 may serve as early markers for human cervical cancer.

The calcium-activated potassium channels KCNMA1 are activated by membrane depolarization, intracellular calcium, and by factors involved in carcinogenesis like hypoxia and growth factors (23). These channels are expressed in many cell types including neurons, smooth muscle, sensory and epithelial cells $(10,24)$, and are over-expressed in different tumor cells and cancers influenced by hormones (12-15). Patch-clamp recordings and the inhibition of cell proliferation in HeLa cells by the KCNMA1 blocker iberiotoxin suggested the presence of these channels in cervical cancer cells (15). Nevertheless, KCNMA1 expression during cervical carcinogenesis, and its expression in human biopsies were unknown.

In this work, we demonstrate first that the in vivo model of transgenic mice is a useful approach to study the expression of ion channels involved in cancer. We observed that the model resembled the in vitro and clinical observations reported for other potassium channels, namely, $\mathrm{KCNH} 1$ channels, which have been suggested as early cervical cancer markers (22). KCNH1 channels are regulated by HPV oncogenes and estradiol, and its protein expression increases from normal cervix to intraepithelial lesions and carcinomas in human biopsies and cervical cytologies $(20,21)$. Here we observed that estradiol increased Kcnhl mRNA and protein expression, and that the highest expression was found in the transgenic mice with cervical carcinoma. Thus, the K14E7 cervical cancer mice are a representative model to study ion channels as early cervical cancer markers.

We also found that the Kcnmal mRNA expression was up-regulated by estradiol in all groups in the in vivo model, but the highest Kcnmal expression was observed in the transgenic mice with cervical cancer. This expression pattern was also observed at the protein level in the immunohistochemistry experiments. The regulation of Kcnmal mRNA levels by estradiol in all groups is interesting because the mouse Kcnmal promoter contains multiple estrogen-responsive sequences (25). On the other hand, $17 \beta$-estradiol binds to the $\beta 1$ and $\beta 4$ subunits of the channel protein inducing its activation (26). It has been also reported that KCNMA1 protein increases during gestation in mouse uterine smooth muscle tissue (27). In addition, pharmacological and knockdown experiments have shown that KCNMA1 channels participate in the estradiol-induced growth in prostate and breast cancer cells $(13,14)$. Kcnmal is also amplified in ovarian and endometrial cancers, which are highly influenced by hormones (13). Thus, our studies suggest the participation of KCNMA1 channels in cervical cell functions associated to estrogens in health and disease, including cervical cancer. Definitely, more studies are needed to elucidate this potential role. Because the KCNMA1 selective blocker iberiotoxin decreases the proliferation of HeLa cervical cancer cells, it would be also very interesting to investigate the effect of iberiotoxin on cervical cancer progression in the in vivo model here studied.

We also observed KCNMA1 protein expression in the endocervical cells of the transformation zone in all groups. However, channel expression in the squamous cells of this zone was only observed in the transgenic mice treated with estrogen, that is, those with either premalignant cervical lesion or cervical cancer. Because the transformation zone is the region where most of the abnormal changes occur in cervical cancer, and where the progenitor cell types for cervical cancer are supposed to reside, these results strongly propose KCNMA1 channels as early cervical cancer markers.

Human cervical biopsies displayed differential KCNMA1 protein expression. While non-cancerous cervix did not show KCNMA1 protein expression, most of the tissues with cervical lesions displayed the presence of the protein. The lowest channel immunostaining intensity was observed in biopsies from low-grade dysplasia and the strongest in the carcinoma tissues. The expression of the channel in low-grade dysplasia, suggests its potential use as an early marker for human cervical cancer. A plausible explanation of KCNMA1 overexpression in human cervical dysplasia and cancer is the regulation of these channels by miRNAs. For instance, mir211 has been proposed as a direct post-transcriptional down-regulator of KCNMA1, because down-regulation of mir211 increases KCNMA1 protein levels in melanoma cells (28). Interestingly, mir211 is down-regulated in cervical cancer (29); this may up-regulate KCNMA1 expression leading to the participation of KCNMA1 channels in cell proliferation and migration, as it has been observed for other cell types (11-14). The regulation of KCNMA1 channel expression in human cervix by cancer-associated factors like HPV oncogenes and estrogens warrants further investigation. In addition, validation studies with more samples to obtain the sensitivity and specificity values of the detection of KCNMA1 channels as a diagnostic tool are needed, as well as more quantitative channel protein analysis, for instance by western blot. Moreover, detection of KCNMA1 channels in a more easy-to-obtain sample (namely, cervical cells from pap-smears or liquid cytology), as well as studies in patients taking estrogens are deserved.

Nevertheless, despite that several clinical and mechanistic studies are needed, our results strongly suggest KCNMA1 channels as potential early markers for human cervical cancer. Detection of this channel in cervical cancer screening programs may help to decrease the mortality from this disease.

\section{Acknowledgements}

This abstract was presented at the 25th World Cancer Conference, October 19-21, 2017 (Rome, Italy) and was published as: ScientificTracks Abstracts: J Cancer Sci Ther 2017, 9:9(Suppl). DOI: 10.4172/1948-5956-C1-111. https://www.omicsonline.org/ conference-proceedings/world-cancer-2017-scientifictracksabstracts.digital/\#25/z.

\section{References}

1. Saslow D, Solomon D, Lawson HW, Killackey M, Kulasingam SL, Cain J, Garcia FA, Moriarty AT, Waxman AG, Wilbur DC, et al: American Cancer Society, American society for colposcopy and cervical pathology and american society for clinical pathology screening guidelines for the prevention and early detection of cervical cancer. Am J Clin Pathol 137: 516-542, 2012.

2. Ferlay J, Soerjomataram I, Ervik M, Dikshit R, Eser S, Mathers C, Rebelo M, Parkin DM, Forman D and Bray F: GLOBOCAN 2012 v1.0. Cancer Incidence and Mortality Worldwide: IARC CancerBase No. 11 [Internet]. IARC, Lyon, 2013. http://globocan. iarc.fr. Accessed August 2, 2017.

3. Au WW, Abdou-Salama S, Sierra-Torres $\mathrm{CH}$ and Al-Hendy A: Environmental risk factors for prevention and molecular intervention of cervical cancer. Int J Hyg Environ Healt 210: 671-678, 2007. 
4. Chung SH, Franceschi S and Lambert PF: Estrogen and ERalpha: Culprits in cervical cancer? Trends Endocrinol Metab 21: 504-511, 2010.

5. Veerapaneni P, Kirma N, Nair HB, Hammes LS, Hall KL and Tekmal RR: Elevated aromatase expression correlates with cervical carcinoma progression. Gynecol Oncol 114: 496-500, 2009.

6. Brake $\mathrm{T}$ and Lambert PF: Estrogen contributes to the onset, persistence and malignant progression of cervical cancer in a human papillomavirus-transgenic mouse model. Proc Natl Acad Sci USA 102: 2490-2455, 2005.

7. Lastraioli E, Iorio J and Arcangeli A: Ion channel expression as promising cancer biomarker. Biochim Biophys Acta 1848 2685-2702, 2015.

8. Pardo LA and Stühmer W: The roles of $\mathrm{K}(+)$ channels in cancer. Nat Rev Cancer 14: 39-48, 2014.

9. Salkoff L, Butler A, Ferreira G, Santi C and Wei A: High-conductance potassium channels of the SLO family. Nat Rev Neurosci 7: 921-931, 2006.

10. Contet C, Goulding SP, Kuljis DA and Barth AL: BK channels in the central nervous system. Int Rev Neurobiol 128: 281-342, 2016.

11. Ge L, Hoa NT, Wilson Z, Arismendi-Morillo G, Kong XT, Tajhya RB, Beeton C and Jadus MR: Big Potassium (BK) ion channels in biology, disease and possible targets for cancer immunotherapy. Int immunopharmacol 22: 427-443, 2014.

12. Liu X, Chang Y, Reinhart PH, Sontheimer H and Chang Y: Cloning and characterization of glioma BK, a novel BK channel isoform highly expressed in human glioma cells. J Neurosci 22 : 1840-1849, 2002.

13. Oeggerli M, Tian Y, Ruiz C, Wijker B, Sauter G, Obermann E, Güth U, Zlobec I, Sausbier M, Kunzelmann K and Bubendorf L: Role of KCNMA1 in breast cancer. PLoS One 7: e41664, 2012.

14. Bloch M, Ousingsawat J, Simon R, Schraml P, Gasser TC, Mihatsch MJ, Kunzelmann K and Bubendorf L: KCNMA1 gene amplification promotes tumor cell proliferation in human prostate cancer. Oncogene 26: 2525-2534, 2007.

15. Han X, Wang F, Yao W, Xing H, Weng D, Song X, Chen G, Xi L, Zhu T, Zhou J, et al: Heat shock proteins and p53 play a critical role in $\mathrm{K}^{+}$channel-mediated tumor cell proliferation and apoptosis. Apoptosis 12: 1837-1846, 2007.

16. Herber R, Liem A, Pitot H and Lambert PF: Squamous epithelial hyperplasia and carcinoma in mice transgenic for the human papillomavirus type 16 E7 oncogene. J Virol 70: 1873-1881, 1996.

17. Riley RR, Duensing S, Brake T, Münger K, Lambert PF and Arbeit JM: Dissection of human papillomavirus E6 and E7 function in transgenic mouse models of cervical carcinogenesis. Cancer Res 63: 4862-4871, 2003.

18. Livak KJ and Schmittgen TD: Analysis of relative gene expression data using real-time quantitative PCR and the 2(-Delta Delta C(T)) method. Methods 25: 402-408, 2001
19. Ibarra Sierra E, Díaz Chávez J, Cortés-Malagón EM, Uribe-Figueroa L, Hidalgo-Miranda A, Lambert PF and Gariglio P: Differential gene expression between skin and cervix induced by the E7 oncoprotein in a transgenic mouse model. Virology 433: 337-345, 2012.

20. Cortés-Malagón EM, Bonilla-Delgado J, Díaz-Chávez J, Hidalgo-Miranda A, Romero-Cordoba S, Uren A, Celik H, McCormick M, Munguía-Moreno JA, Ibarra-Sierra E, et al: Gene expression profile regulated by the HPV16 E7 oncoprotein and estradiol in cervical tissue. Virology 447: 155-165, 2013.

21. Díaz L, Ceja-Ochoa I, Restrepo-Angulo I, Larrea F, Ávila-Chávez E, García-Becerra R, Borja-Cacho E, Barrera D, Ahumada E, Gariglio P, et al: Estrogens and human papilloma virus oncogenes regulate human ether-à-go-go-1 potassium channel expression. Cancer Res 69: 3300-3307, 2009.

22. Ortiz CS, Montante-Montes D, Saqui-Salces M, Hinojosa LM, Gamboa-Domínguez A, Hernández-Gallegos E, Martínez-Benítez B, Del Rosario Solís-Pancoatl M, Garcia-Villa E, Ramírez A, et al: Eag1 potassium channels as markers of cervical dysplasia. Oncol Rep 26: 1377-1383, 2011.

23. Bentzen BH, Olesen SP, Rønn LC and Grunnet M: BK channel activators and their therapeutic perspectives. Front Physiol 5: 389, 2014.

24. McCobb DP, Fowler NL, Featherstone T, Lingle CJ, Saito M, Krause JE and Salkoff L: A human calcium-activated potassium channel gene expressed in vascular smooth muscle. Am J Physiol 269: H767-H777, 1995.

25. Kundu P, Alioua A, Stefani E and Toro L: Regulation of mouse Slo gene expression: Multiple promoters, transcription start sites and genomic action of estrogen. J Biol Chem 282: 27478-27492, 2007.

26. Valverde MA, Rojas P, Amigo J, Cosmelli D, Orio P, Bahamonde MI, Mann GE, Vergara C and Latorre R: Acute activation of maxi-K channels (hSlo) by estradiol binding to the $\beta$ subunit. Science 285: 1929-1931, 1999.

27. Benkusky NA, Fergus DJ, Zucchero TM and England SK: Regulation of the $\mathrm{Ca}_{2}+$-sensitive domains of the maxi-K channel in the mouse myometrium during gestation. J Biol Chem 275: 27712-27719, 2000.

28. Mazar J, DeYoung K, Khaitan D, Meister E, Almodovar A, Goydos J, Ray A and Perera RJ: The regulation of miRNA-211 expression and its role in melanoma cell invasiveness. PLoS One 5: e13779, 2010.

29. Tang T, Wong HK, Gu W, Yu MY, To KF, Wang CC, Wong YF, Cheung TH, Chung TK and Choy KW: MicroRNA-182 plays an onco-miRNA role in cervical cancer. Gynecol Oncol 129: 199-208, 2013. 